\title{
PRESENCIA DE MICROORGANISMOS EN TELÉFONOS MÓVILES DEL PERSONAL DE CUIDADOS INTENSIVOS DE UN HOSPITAL DE ESPAÑA
}

\author{
Yeray Gabriel Santana-Padilla 1,a , Luciano Santana-Cabrera ${ }^{1, b}$, María Elena Dorta-Hung ${ }^{1, c}$, \\ Manuel Jesús Molina-Cabrillana ${ }^{1, d}$
}

\begin{abstract}
RESUMEN
Con el objetivo de identificar la presencia de microorganismos en los teléfonos móviles del personal de la unidad de cuidados intensivos $(\mathrm{UCI})$ de un hospital en España se realizó un estudio transversal descriptivo entre los profesionales sanitarios y no sanitarios que tuvieran teléfonos móviles durante su jornada laboral. La recogida de muestras se llevó a cabo mediante hisopo y siembra en placa de cultivo con análisis e identificación de los microorganismos. Se analizaron 111 teléfonos móviles, de los cuales 56 estaban contaminados siendo $41,5 \%$ y $41,1 \%$ para los que pertenecían a las enfermeras y los médicos respectivamente y $60,6 \%$ para la encontrada en los auxiliares de enfermería, destaca el crecimiento de Pseudomonas aeruginosa (12,5\%), Staphylococus aureus meticilin-resistente $(10,9 \%)$, y Stenotrophomonas maltophila (4,7\%). En conclusión, los teléfonos móviles presentaron presencia de microorganismos de relevancia clínica. La presencia de estas bacterias y hongos deben alertarnos sobre la posibilidad de que sirvan de reservorio para la transmisión al paciente crítico.
\end{abstract}

Palabras Claves: Contaminación de Equipos; Prevención de Enfermedades, Teléfono Celular; Personal de Salud; Transmisión, Cuidados Intensivos. (fuente: DeCS BIREME).

\section{PRESENCE OF MICROORGANISMS IN MOBILE PHONES OF INTENSIVE CARE STAFF AT A HOSPITAL IN SPAIN}

\begin{abstract}
In order to identify the presence of microorganisms in mobile phones of the staff from the intensive care unit (ICU) at a hospital in Spain, a cross-sectional descriptive study was carried out among healthcare and non-healthcare professionals who had mobile phones during their working day. Samples were collected by swabbing and sowing on a culture plate with analysis and identification of microorganisms. One hundred eleven (111) mobile phones were analyzed, of which 56 were contaminated: $41.5 \%$ and $41.1 \%$ of those belonging to nurses and physicians, respectively, and $60.6 \%$ found in nursing assistants. The growth of Pseudomonas aeruginosa (12.5\%), methicillin-resistant Staphylococcus aureus $(10.9 \%)$, and Stenotrophomonas maltophila (4.7\%) stand out. In conclusion, mobile phones presented the presence of clinically relevant microorganisms.
\end{abstract}

Keywords: Equipment Contamination; Disease Prevention; Cell phone; Health Personnel; Transmission; Critical Care. (source: MeSH NLM).

\section{INTRODUCCIÓN}

Dentro de los sistemas sanitarios, un problema que alarma especialmente a los profesionales son las infecciones asociadas a la asistencia sanitaria. Esto ha merecido el desarrollo de múltiples medidas preventivas para evitar la propagación de diversos microorganismos, siendo la más eficaz, eficiente y efectiva la higiene de manos ${ }^{(1-3)}$. Los dispositivos móviles, como los teléfonos, son elementos indispensables para la comunicación. Sin embargo, sin

Hospital Universitario Insular de Gran Canaria. Las Palmas de Gran Canaria. España.

Enfermero; master en ciencias de la enfermería; ${ }^{b}$ médico especialista en medicina intensiva; doctor en ciencias médicas; ${ }^{\mathrm{c}}$ médico especialista en microbiología y parasitología; doctora en medicina; ${ }^{\mathrm{d}}$ médico especialista en medicina preventiva; doctor en Medicina

Recibido: 30/03/2019 Aprobado: 06/11/2019 En línea: 03/12/2019

Citar como: Santana-Padilla YG, Santana-Cabrera L, Dorta-Hung ME, Molina-Cabrillana MJ. Presencia de microorganismos en teléfonos móviles del personal de cuidados intensivos de un hospital de España. Rev Peru Med Exp Salud Publica. 2019;36(4):676-80. doi: 10.17843/rpmesp.2019.364.4421. 
una adecuada higiene, podría actuar como un reservorio para la transmisión de patógenos, encontrando datos muy variables en cuanto a los microorganismos detectados ${ }^{(4-9)}$.

Existen diversas teorías al respecto de por qué estos dispositivos son vectores de patógenos, estos equipos generan condiciones para el crecimiento de microorganismos que tenemos en nuestras manos y que llegan allí al manipularlos sin una adecuada higiene ${ }^{(10,11)}$, también se ha encontrado en las manos del personal de salud y en los teléfonos móviles abundante cantidad de bacterias potencialmente patógenas en el ámbito nosocomial ${ }^{(12-15)}$.

Por lo expuesto el objetivo de este estudio es identificar la presencia de microorganismos en los teléfonos móviles del personal de una unidad de cuidados intensivos.

\section{EL ESTUDIO}

Se realizó un estudio transversal descriptivo seleccionando los teléfonos móviles por cada personal de salud durante noviembre de 2017 y abril de 2018. El ámbito de estudio fue la unidad de cuidados intensivos perteneciente a un hospital de tercer nivel que cuenta con 24 camas y cerca de 1100 ingresos al año (2017), con un índice de ocupación del $70 \%$ y una estancia media de $7,51 \pm 12,39$ días. El personal adscrito a la unidad son 26 médicos (incluidos los médicos residentes), 70 enfermeras (una enfermera por dos pacientes) y 50 auxiliares de enfermería (un auxiliar de enfermería por tres pacientes). Además de un fisioterapeuta y doce celadores. Estos profesionales tienen un índice de adhesión al programa de higiene de manos inferior al $50 \%$ durante el período que se llevó a cabo el estudio, detectado mediante observación directa por la unidad de medicina preventiva. La unidad tiene además programas de uso prudente y rotación de antibióticos.

Se incluyeron profesionales sanitarios y no sanitarios que disponían de teléfonos móviles durante su jornada laboral, constituyéndose en un muestreo por conveniencia. En el presente estudio los profesionales sanitarios enrolados fueron médicos, incluidos residentes, enfermeras, auxiliares de enfermería y fisioterapeutas; los profesionales no sanitarios incluidos en el presente estudio fueron los celadores.

La recogida de muestras de los teléfonos móviles se realizó mediante hisopo. Se siguió el procedimiento aprobado por el servicio de medicina preventiva del hospital para la recogida de muestra de superficie mediante hisopo recolector de muestras con tubo y medio de transporte (MDD C€ 0318 Deltalab®). El procedimiento constaba de los siguientes pasos. En primer lugar, se utilizó un

\section{MENSAJES CLAVE}

Motivación para realizar el estudio. Las unidades de cuidados intensivos por sus especiales características son áreas de especial consideración relacionadas con las enfermedades nosocomiales.

Principales hallazgos. 111 dispositivos móviles fueron analizados de las cuales el 49,5\% Los organismos identificados fueron Streptococcus spp (21), Bacillus spp (10), Corynebacterium spp (8), Staphylococus aureus meticilina resistente (7), Pseudomonas Aeruginosa (8), Stenotrophomonas maltophilia (3), Pantoea aglomerans (1) y Candidas spp (6).

Implicancias. El aislamiento de microorganismos patógenos en estos dispositivos debe alertarnos de que pueden servir como reservorio de transmisión hacia el enfermo crítico.

hisopo estéril humedecido con medio BHI (infusión cerebro corazón). Una vez humedecido se frota el hisopo en la superficie a estudiar, por lo menos en dos direcciones distintas y rotándolo ligeramente, recorriendo toda la superficie de la pantalla del móvil. Posteriormente se corta la cabeza del hisopo con tijeras estériles para depositarlo dentro del tubo de BHI. Se identifica la muestra con un número único y se envía al laboratorio a temperatura ambiente, inmediatamente después de la toma de muestras. Se introduce en una estufa destinada a tal efecto, donde se procederá a su siembra a las 24-48 horas en tres medios (agar sangre, agar McConkey y agar infusión cerebro corazón) y su interpretación a los cinco a seis días. Para finalizar, se limpia cuidadosamente con solución desinfectante (alcohol $70 \%$ ) el área muestreada con el fin de eliminar los restos de medio de cultivo ya que suelen quedar restos del medio de cultivo utilizado que puedan favorecer el sobrecrecimiento de microorganismos en esa zona.

La recogida y procesamiento de la muestra fue siempre llevado a cabo por la misma enfermera de $\mathrm{UCI}$, que fue formada específicamente por el servicio de medicina preventiva para la toma de muestras en superficie, según los protocolos aprobados en la propia institución. La médico microbióloga del servicio de medicina preventiva fue quien analizó, sembró todos los hisopos analizados e interpretó los hallazgos microbiológicos.

Los datos se procesaron como frecuencias y porcentajes y se analizaron en el programa estadístico SPSS 15.0. El estudio contó con la aprobación de la Unidad de Formación Continuada, Docencia e Investigación del Complejo Hospitalario. El propósito de la investigación fue explicado a los participantes y el consentimiento informado verbal fue obtenido antes de la toma de muestras en sus teléfonos móviles. 


\section{HALLAZGOS}

Un total de 111 teléfonos móviles fueron analizados; esta muestra representa el $69,8 \%$ del total de trabajadores de la UCI. Según los participantes el $65,4 \%$ fueron médicos, el $75,7 \%$ enfermeras, el $66 \%$ auxiliares de enfermería, el $100 \%$ de los fisioterapeutas y el $58,3 \%$ celadores.

Se detectaron un total de 56 muestras contaminadas $(49,5 \%)$, con la detección de 64 determinaciones de microorganismos. Existían ocho muestras con dos microorganismos detectados, la combinación más frecuente de estas muestras fueron la colonización por StreptococcusCandida con el $50 \%$. Por estamentos, las enfermeras y los médicos tenían una frecuencia de contaminación, en sus dispositivos de $41,5 \%$ y $41,1 \%$ respectivamente, siendo para el grupo de auxiliares del $60,6 \%$.

De los 64 microorganismos aislados en los teléfonos móviles, los Gram positivos fueron el 71,9\%, los Gram negativos el $18,8 \%$ y los hongos el 9,4\%. La frecuencia de microorganismos en cada grupo estuvo constituida de la siguiente manera: Gram (+) Streptococcus spp (21), Bacillus spp (10), Corynebacterium spp (8), Staphylococus aureus meticilina resistente (7); Gram (-) Pseudomonas Aeruginosa (8), Stenotrophomonas maltophilia (3), Pantoea aglomerans (1) y Candida spp (6).

La Tabla 1 muestra los microorganismos aislados, en función del estamento laboral, destacando que de las seis muestras aisladas de Candida spp., tres pertenecian a médicos, lo que representa un $50 \%$ de las muestras con hongos. Sin embargo, en las enfermeras y auxiliares de enfermería predominaban la presencia de Gram positivos.

En la Tabla 2 se puede observar los diferentes microorganismos aislados, en los teléfonos móviles en los diferentes grupos. Destaca la presencia de microorganismos del tipo de Staphylococus aureus meticilina resistente (10,9\%), Pseudomonas aeruginosa (12,5\%) y Stenotrophomonas maltophilia (4,7\%).

Tabla 1. Tipos de microorganismos aislados en los teléfonos móviles en función del estamento laboral

\begin{tabular}{lccc}
\hline Grupo & $\begin{array}{c}\text { Gram } \\
\text { negativos }\end{array}$ & $\begin{array}{c}\text { Gram } \\
\text { positivos }\end{array}$ & Hongos \\
\hline Médicos & 2 & 7 & 3 \\
Enfermeras & 6 & 18 & 1 \\
Auxiliares de Enfermería & 4 & 15 & 1 \\
Celadores & 0 & 5 & 1 \\
Fisioterapeuta & 0 & 1 & 0 \\
Total & 12 & 46 & 6 \\
\hline
\end{tabular}

\section{DISCUSIÓN}

En nuestro estudio encontramos que la mitad de los teléfonos presentaron contaminación. Cifras elevadas pero que no alcanzan las mostradas por otros autores como Ulger y cols ${ }^{(5)}$ que alcanzaban hasta el $94,5 \%$ o Jayalakshmi y cols ${ }^{(16)}$ con el $91,6 \%$. Sin embargo, estudios como el de Smibert y cols ${ }^{(17)}$ encontraron una contaminación del $7 \%$ de organismos multirresistentes implicando una baja evidencia, según estos autores, para el establecimiento de protocolos de limpieza para estos dispositivos en las $\mathrm{UCl}$ estudiadas.

Es de resaltar el aislamiento de microorganismos multirresistentes en las muestras recogidas. Así, en casi $11 \%$ de las muestras, crecía el Staphylococcus aureus resistente a meticilina (SAMR), a pesar de que ninguno de los pacientes ingresados durante el período del estudio era portador de dicho patógeno. Los reservorios de SAMR son principalmente los pacientes pero el personal sanitario, que también puede estar colonizado, puede colaborar en la transmisión que se produce fundamentalmente de forma cruzada a través de las manos, aunque no debemos olvidar el rol que desempeña el ambiente hospitalario (superficies, material de uso clínico, etc.) ${ }^{(18)}$. Por tanto, podemos valorar que puede existir un reservorio de SARM entre los trabajadores, lo cual debe ser analizado mediante el muestreo nasal.

La presencia de microorganismos multirresistentes del tipo de Pseudomonas aeruginosa o Stenotrophomonas, podría justificar una transmisión cruzada entre el personal. Además, hay que tener en cuenta que puede producirse contaminación de equipos hospitalarios o teléfonos móviles, en particular de aquellos que poseen componentes en contacto con la humedad ${ }^{(19)}$.

En cuanto a las medidas de prevención de la transmisión de microorganismos entre los pacientes hospitalizados, uno de los aspectos relevantes a tener en cuenta es la contaminación ambiental como factor implicado en la diseminación de los microorganismos en los hospitales ${ }^{(20)}$. Los microorganismos detectados son de relevancia clínica por lo que se debe hacer una serie de recomendaciones generales como son la limpieza de superficies y desinfección adecuada de los teléfonos móviles al finalizar la jornada, y hacer hincapié a la higiene de manos antes de tocar los propios teléfonos ${ }^{(7)}$. Actualmente no existe protocolo de limpieza de teléfonos móviles ni restricciones de su uso pues no se consideraba un material de uso hospitalario, pero ahora nos planteamos la elaboración de un protocolo de limpieza para esta superficie. Estudios como el realizado por Smibert ${ }^{(17)}$ muestran una alta adherencia en el lavado de manos, por lo que se debe incidir en esta práctica para 
Tabla 2. Microorganismos aislados en los teléfonos móviles según estamento laboral

\begin{tabular}{lccccc}
\hline Microorganismo & $\begin{array}{c}\text { Médicos } \\
\mathbf{n ~ ( \% )}\end{array}$ & $\begin{array}{c}\text { Enfermeras } \\
\mathbf{n}(\%)\end{array}$ & $\begin{array}{c}\text { Auxiliares de } \\
\text { Enfermería } \mathbf{n}(\%)\end{array}$ & $\begin{array}{c}\text { Celadores } \\
\mathbf{n}(\%)\end{array}$ & $\begin{array}{c}\text { Fisioterapeuta } \\
\mathbf{n}(\%)\end{array}$ \\
\hline Pantoea aglomerans & 0 & 0 & $1(1,5)$ & 0 & 0 \\
Staphylococus aureus MR & 0 & $2(3,1)$ & $4(6,2)$ & $1(1,5)$ & 0 \\
Bacillus spp. & $1(1,5)$ & $7(10,9)$ & $1(1,5)$ & $1(1,5)$ & 0 \\
Corynebacterium spp. & 0 & $4(6,2)$ & $3(4,6)$ & 0 & $1(1,5)$ \\
Pseudomonas aeruginosa & $1(1,5)$ & $5(7,8)$ & $2(3,1)$ & 0 & 0 \\
Streptococcus spp. & $6(9,3)$ & $5(7,8)$ & $7(10,9)$ & $3(4,6)$ & 0 \\
Stenotrophomonas maltophilia & $1(1,5)$ & $1(1,5)$ & $1(1,5)$ & 0 & 0 \\
Candida spp. & $3(4,6)$ & $1(1,5)$ & $1(1,5)$ & $1(1,5)$ & 0 \\
\hline
\end{tabular}

MR: Meticilina resistente

disminuir también las contaminaciones de los teléfonos móviles.

El presente estudio tiene una serie de limitaciones. En primer lugar, no se ha realizado un muestreo aleatorio por lo que las estimaciones pudieran estar sesgadas; no obstante, se ha analizado a más de la mitad del personal de esta unidad. La segunda limitación es que no se ha realizado análisis molecular para valorar la identidad clonal de las cepas aisladas en los teléfonos móviles y las de los pacientes. No nos ha parecido oportuno al ser una técnica costosa y no poder usarla de manera habitual en nuestro medio.

En conclusión, en el presente estudio se han detectado microorganismos en los teléfonos móviles; su sola presencia permite alertar a que pudieran servir como reservorio de transmisión hacia el enfermo crítico. La ausencia de costumbre de limpiar los teléfonos móviles debiera ser estudiada siendo necesario insistir en la higiene de manos para prevenir la dispersión de microorganismos en el ambiente hospitalario. Estos hallazgos obligan a incentivar las medidas educacionales higiénicas en todos los estamentos y sobre todo con el personal auxiliar de enfermería, donde el aislamiento de microorganismos fue importante, asimismo proponer la puesta en marcha de un protocolo de limpieza de teléfonos móviles.

\section{Fuentes de financiamiento: Autofinanciado}

Conflictos de intereses: Ninguno

Contribuciones de autoría: YGSP, LSC, EMDH y MJMC han participado en la concepción y diseño del artículo, interpretación de dato, redacción y revisión crítica del artículo. YGSP participó en la recolección de muestras. EMDH participó en la asesoría técnica y administrativa. Todos los autores aprobaron la versión final.

\section{REFERENCIAS BIBLIOGRÁFICAS}

1. Tacconelli E, Cataldo MA, Dancer SJ, De Angelis G, Falcone M, Frank $\mathrm{U}$, et al. ESCMID guidelines for the management of the infection control measures to reduce transmission of multidrug-resistant Gram-negative bacteria in hospitalized patients. Clin Microbiol Infect. 2014;20:1-55. doi: 10.1111/1469-0691.12427

2. Allegranzi B, Gayet-Ageron A, Damani N, Bengaly L, McLaws M-L, Moro M-L, et al. Global implementation of WHO's multimodal strategy for improvement of hand hygiene: a quasi-experimental study. Lancet Infect Dis. 2013;13(10):843-51. doi: 10.1016/S1473-3099(13)70163-4

3. Adegboye MB, Zakari S, Ahmed BA, Olufemi GH. Knowledge, awareness and practice of infection control by health care workers in the intensive care units of a tertiary hospital in Nigeria. Afr Health Sci. 2018;18(1):72-78. doi: 10.4314/ahs. v18i1.11

4. Pal S, Juyal D, Adekhandi S, Sharma M, Prakash R, Sharma N, et al. Mobile phones: Reservoirs for the transmission of nosocomial pathogens. Adv Biomed Res. 2015;4(1):144. doi: 10.4103/22779175.161553

5. Ulger F, Dilek A, Esen S, Sunbul M, Leblebicioglu H. Are healthcare workers' mobile phones a potential source of nosocomial infections.Review of the literature. J Infect Dev Ctries. 2015;9(10):1046-53. doi: 10.3855/ jidc.6104

6. Kotris I,DrenjančevićD, TalapkoJ, Bukovski S. Identification of microorganisms on mobile phones of intensive care unit health care workers and medical students in the tertiary hospital. Med Glas (Zenica). 2017;14(1):85-90. doi: 10.17392/878-16

7. Heyba M, Ismaiel M, Alotaibi A, Mahmoud M, Baqer H, Safar A, et al. Microbiological contamination of mobile phones of clinicians in intensive care units and neonatal care units in publichospitals in Kuwait. BMC Infect Dis. 2015;15(1):434. doi: 10.1186/s12879-015-1172-9

8. Nwankwo EO, Ekwunife N, Mofolorunsho KC. Nosocomial pathogens associated with the mobile phones of healthcare workers in a hospital in Anyigba, Kogi state, Nigeria. J Epidemiol Glob Health. 2014;4(2):13540. doi: 10.1016/j.jegh.2013.11.002 
9. Kordecka A, Krajewska-Kułak E, Łukaszuk C, Kraszyńska B, Kułak W. Isolation frequency of Candida present on the surfaces of mobile phones and handsx. BMC Infect Dis. 2016;16(1):238. doi: 10.1186/s12879016-1577-0

10. Ulger F, Esen S, Dilek A, Yanik K, Gunaydin M, Leblebicioglu $\mathrm{H}$. Are we aware how contaminated our mobile phones with nosocomial pathogens. Ann Clin Microbiol Antimicrob. 2009;8(1):7. doi: 10.1186/14760711-8-7

11. Foong YC, Green M, Ogden K. Mobile phones as a potential vector of infection in a paediatric ward. J Paediatr Child Health. 2013;49(12):1083-4. doi: $10.1111 /$ jpc. 12438

12. Loyola S, Gutierrez LR, Horna G, Petersen K, Agapito J, Osada J, et al. Extended-spectrum $\beta$-lactamase-producing Enterobacteriaceae in cell phones of health care workers from Peruvian pediatric and neonatal intensive care units. Am J Infect Control. 2016;44(8):910-6. doi:10.1016/j.ajic.2016.02.020
13. Loyola S, Gutierrez L, Avendaño E, Severino N, Tamariz J. Multidrugresistant bacteria isolated from cell phones in five intensive care units: Exploratory dispersion analysis. Germs. 2018;8(2):8591. doi: 10.18683/germs.2018.1135

14. Russotto V, Cortegiani A, Raineri SM, Giarratano A. Bacterial contamination of inanimate surfaces and equipment in the intensive care unit. J Intensive Care. 2015;3(1):54. doi: 10.1186/s40560015-0120-5

15. Khan A, Rao A, Reyes-Sacin C, Hayakawa K, Szpunar S, Riederer K, et al. Use of portable electronic devices in a hospital setting and their potential for bacterial colonization. Am J Infect Control. 2015;43(3):286-8. doi: 10.1016/j. ajic.2014.11.013

16. Jayalakshmi J, Appalaraju B, Usha S. Cellphones as reservoirs of nosocomial pathogens. J Assoc Physicians India. 2008;56:388-9. PMID: 18700649

17. Smibert OC, Aung AK, Woolnough E, Carter GP, Schultz MB, Howden BP, et al. Mobile phones and computer keyboards: unlikely reservoirs of multidrug-resistant organisms in the tertiary intensive care unit. J Hosp Infect. 2018;99(3):295-8. doi: 10.1016/j.jhin.2018.02.013

18. Verbrugh H. Preventing nosocomial MRSA infections by screening. Euro Surveill. 2014;19(37).

19. Cobos-Trigueros N, Solé M, Castro P, Torres JL, Hernández C, Rinaudo $\mathrm{M}$, et al. Acquisition of Pseudomonas aeruginosa and its resistance phenotypes in critically ill medical patients: role of colonization pressure and antibiotic exposure. Crit Care. 2015;19(1):218. doi: 10.1186/s13054-015-0916-7

20. Lupión C, López-Cortés LE, RodríguezBaño J. Preventive measures for avoiding transmission of microorganisms between hospitalised patients. Handhygiene.Enferm Infecc Microbiol Clin. 2014;32(9):603-9. doi: 10.1016/j.eimc.2014.02.003

Correspondencia: Yeray Gabriel SantanaPadilla.

Dirección: Hospital Universitario Insular de Gran Canaria. Avenida Maritima del Sur, s/n. Las Palmas de Gran Canaria. Islas Canarias, España 35016.

Correo electrónico: yeraysantana@celp.es 